\title{
Fluxes in M Giants With Improved Water Vapor Opacity
}

\author{
D.R. Alexander \\ Wichita State University \\ J.A. Brown \\ G.C. Augason \\ NASA/Ames Research Center \\ Indiana University
}

In the atmospheres of cool oxygen-rich stars, water vapor is the most important molecular absorber. Yet no laboratory or theoretical spectrum exists for water vapor of sufficient detail to be useful for radiative transfer calculations in a stellar atmosphere. Because of the complexities of its bent triatomic structure, this deficiency is not likely to the be resolved soon. We have, therefore, undertaken the preparation of a synthetic spectrum for water vapor.

For this synthesis, we produce hypothetical lines of water vapor which accurately reproduce narrow-band laboratory observations. The calculations are based upon the laboratory observations of Ludwig, C.B.. et al. (NASA SP-3080, 1973), for the determination of straight mean opacities and mean line spacings, and Rothman, L.S., et al. (AFCRL, private communication, 1987), for the determination of the distribution of energy levels in the hot water vapor spectrum. Lines are placed randomly in intervals determined by the mean line spacing. Their strengths are taken randomly according to an exponential distribution to reproduce the observed straight mean for that interval. The lower energy level for each line is determined randomly from the distribution of lower energy levels for lines of similar strength and wavelength.

The resulting spectrum contains over four and a half million lines. The average opacity computed from this synthetic spectrum accurately reproduces the laboratory data. Altogether, our line archive for the computation of model stellar atmospheres now contains nearly 21 million lines. Compared to our previous models, which included only straight mean opacities for water vapor, these models have, generally lower opacities, higher temperatures, and higher pressures over most of the atmospheres, with a reversal of that trend in the very shallowest layers.

Emergent flux curves for models calculated using the new opacities lack the large-scale discontinuities caused by the stop-wise nature of the straight-mean opacities seen in previous flux curves. The $\mathrm{H}^{-}$flux peak at $1.65 \mu \mathrm{m}$ is greatly reduced due to a correction in the calculation of the $\mathrm{H}^{-}$bound-free opacity. The strength of the water vapor bands is also diminished. While the models fit observations better than previous ones, they show marginally too strong TiO features, insufficient opacity around $1 \mu \mathrm{m}$, and too much visual flux. 